\title{
MEMS Pirani type vacuum sensor with extended sensitivity range
}

\author{
F. Völklein**, C. Dütsch*, M.Grau**, J. Schieferdecker*, M. Simon*, K. Storck* \\ * Heimann Sensor GmbH, Grenzstraße 22, D-01109 Dresden; \\ ** RheinMain University of Applied Sciences, Institute for Microtechnologies (IMtech), Am Brückweg 26, \\ 65428 Rüsselsheim
}

\begin{abstract}
Heimann Sensor together with RheinMain University of Applied Sciences have developed a new vacuum sensor with an extended measuring range. While most of conventional and MEMS Pirani type vacuum gauges detect pressures between $10^{3}$ mbar and $10^{-3}$ mbar [1-8], the new sensor extends the measuring range down to $10^{-5} \mathrm{mbar}$. By using thermal simulations a chip has been designed where the nonpressure-dependent thermal conductance is significantly reduced. The results of sensor characterization are presented. A modified chip has been specially designed for measurements in the range from $10^{3}$ mbar to 1 mbar. By integrating both chips in the same housing we provide a Pirani type vacuum sensor that shows a pressure-dependent signal from $10^{3} \mathrm{mbar}$ down to $10^{-5} \mathrm{mbar}$.
\end{abstract}

\section{Introduction}

Several years ago IMtech together with Heimann Sensor introduced miniaturized Pirani type vacuum sensors. These thermal sensors use a thin film heater/sensor structure. Its temperature increase depends on the thermal conductance of the surrounding gas and therefore on gas pressure. The temperature dependent resistor is patterned by microlithography of a metal film with high temperature coefficient. It is deposited on a thin membrane layer that has been fabricated by silicon micromachining on a silicon chip. Such miniaturized vacuum sensors open new application fields because of their small size and power consumption. However, for some of these applications an improved measuring sensitivity below $10^{-3}$ mbar is required. This improvement has been achieved by an optimized chip design and advanced fabrication technologies.

\section{Thermal Model and Simulation}

For the thermal modelling the membrane with the deposited thin film resistor (Fig 1) is considered as an isothermal heated area $A$ with pressure-dependent temperature increase $T_{p}-T_{0}$ related to the ambient temperature $\left(T_{0}\right)$. Heat flux occurs from the heated area to the surroundings (heat sinks) via thermal conduction through membrane and metal connection lines $\left(G_{L}\right)$, via radiation from the membrane surfaces $\left(G_{S}\right)$ and via pressure-dependent thermal conduction through the ambient gas $G(p)$, resulting in a total thermal conductance $\mathrm{G}$ :

$$
G=G_{L}+G_{S}+G(p)
$$

For a sensitivity increase at low pressures we have to accomplish $G_{L}$ and $G_{S}$ as small as possible. $G_{L}$ can be decreased by reducing the cross sections of the resistor metal lines and by using a membrane that is suspended on 4 cantilevers instead of a fully supported closed membrane. $G_{S}$ can be reduced by a decreased membrane area and by reducing its emissivity. But since $G(p)$ also scales with the area its optimum size has to be identified by using specific design rules [9]. The reduction of emissivity has been achieved by deposition of metal layers on both sides of the membrane. An additional insulating layer between the heating/sensing resistor and the reflecting layer avoid electrical short-circuits of the resistor.

$G(p)$ can be approximated by the sum of two conducting gas columns of cross section $A$ and length $d_{1}$ (distance between membrane and chip header) and $d_{2}$ (distance between membrane and housing), respectively.

$$
G(p) \propto \lambda_{\text {gas }}\left(p, d_{1}\right) A / d_{1}+\lambda_{\text {gas }}\left(p, d_{2}\right) A / d_{2}
$$


The thermal conductivities of the gas $\lambda_{\text {gas }}\left(p, d_{1}\right)$ and $\lambda_{\text {gas }}\left(p, d_{2}\right)$, respectively, depend on specific gas parameters, the pressure-dependent mean free path of the gas molecules and the distances $d_{1}, d_{2}$ between the membrane and ambient surfaces of temperature $T_{0}$. For low pressures $\left(\operatorname{pd}_{1}, \operatorname{pd}_{2}<10^{-5} \mathrm{~m}\right.$. mbar) $\lambda_{\text {gas }}\left(p, d_{1}\right)$ and $\lambda_{\text {gas }}\left(p, d_{2}\right)$ become directly proportional to $\mathrm{pd}_{1}$ and $\mathrm{pd}_{2}$, respectively, which results in:

$$
G(p) \propto A p
$$

\section{Sensor Design}

Using the results of FEM simulations a chip with suspended membrane and optimized geometrical dimensions has been designed. Two compensation resistors $R_{K}\left(T_{0}\right)$ are patterned besides the heating /sensing resistor $R_{P}\left(T_{p}\right)$ on the bulk silicon rim of the chip.

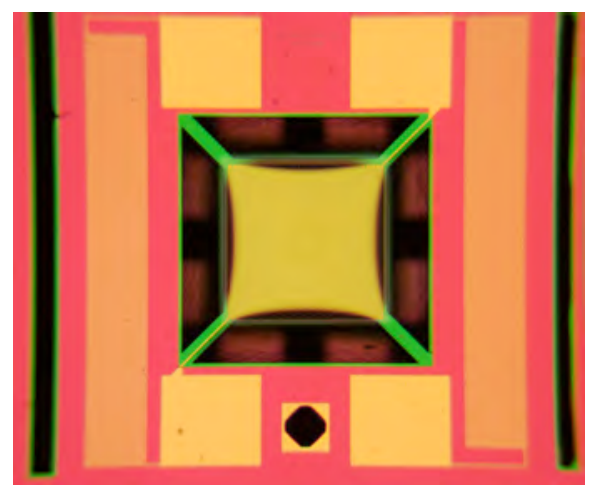

Fig. 1: Sensor chip with thin film resistor on a suspended membrane and two compensation resistors

The recommended readout for the vacuum sensor by using a Wheatstone bridge circuit with constant bridge voltage supply $U_{B}$ is shown in Figure 2 .

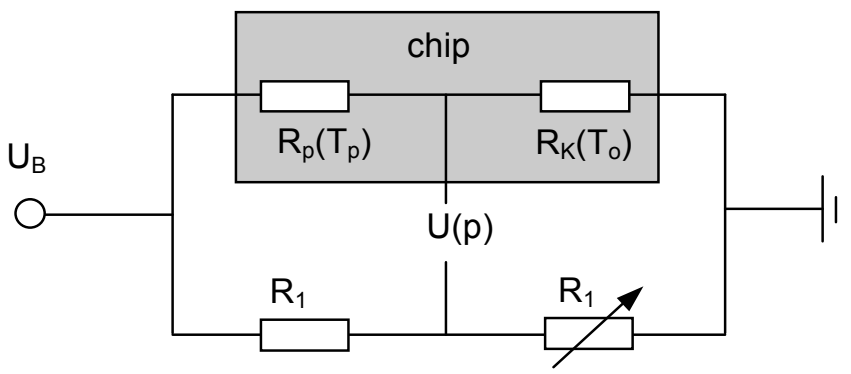

Fig. 2: Wheatstone bridge circuit for readout of the vacuum sensor

The signal voltage $U(p)$ depends on the bridge voltage supply $U_{B}$ and the relative resistance change $\Delta R_{P} / R_{P}$ of the resistor $R_{P}\left(T_{p}\right)$.

$$
U(p) \approx U_{B} \Delta R_{P} / 4 R_{P} \approx U_{B} ß\left(T_{p}-T_{0}\right) / 4
$$

Resistors with large temperature coefficients $ß$ generate higher signal variations than resistors with small ß-values. Therefore, Nickel films with $\beta$ up to $5.5 \cdot 10^{-3} / \mathrm{K}$ are used as thin film resistors.

\section{Thermal Conductances}

After chip fabrication on 4-inch (100) silicon wafers and chip separation, a micromachined silicon bridge is placed on the top side of the chip (with distance $\mathrm{d}_{2}=50 \mu \mathrm{m}$ between membrane and silicon bridge). The sensor parameters, presented in this chapter, are determined on chips mounted on headers and covered with such silicon bridges. The temperature coefficient of the applied Ni film was determined by evaluating the resistance-temperature curve $R_{P}\left(T_{0}\right)$. Between room temperature and $80{ }^{\circ} \mathrm{C}$ it can be approximated 
by a linear characteristic but for a wider temperature range the curve shows a non-linear behaviour. Since $\mathrm{G}(\mathrm{p})$ can be neglected in the UHV-range, measurements of the conductances $G_{L}$ plus $G_{S}$ are performed at vacuum pressures close to $10^{-7}$ mbar. The resistor is heated with power $\mathrm{N}$ and the temperature increase $T-T_{0}$ of the membrane is determined by using the $R_{P}\left(T_{0}\right)$ curve of the heating/sensing resistor. Then, the thermal conductance can be calculated by

$$
\mathrm{G}=\mathrm{N} /\left(\mathrm{T}-\mathrm{T}_{\mathrm{O}}\right)
$$

By measuring $G=G_{L}+G_{S}$ at UHV conditions and calculating $G_{L}$ by using the thermal conductivities of the $\mathrm{Ni}$ film and membrane material, respectively, the thermal conductances have been evaluated.

The formerly offered chip design VAC-01 with fully supported closed membrane suffers from a large thermal conductance $G_{L}$ and, therefore, low sensitivity below $10^{-3}$ mbar. The new redesigned chips VAC02 and VAC-03 are distinct with respect to membrane area A and shape of the 4 cantilevers. The measured thermal conductances are summarized in Table 1. These values demonstrate a significant reduction especially for $G_{L}$. Since $G_{L}$ and $G_{S}$ are now in the same order of magnitude the further decrease of $G_{s}$ becomes more important. Both terms have been reduced in comparison to $G(p)$ thus increasing the pressure sensitivity of the chip.

Table 1: Thermal conductances of various chip designs VAC-1, VAC-2 and VAC-3, respectively

\begin{tabular}{|l|l|l|l|}
\hline Chip type & Membrane area $\mathbf{A}\left(\mathbf{m m}^{\mathbf{2}}\right)$ & $\mathbf{G}_{\mathbf{L}}\left(\mathbf{1 0 ^ { - 6 }} \mathbf{W} / \mathbf{K}\right)$ & $\mathbf{G}_{\mathbf{s}}\left(\mathbf{1 0 ^ { - 6 }} \mathbf{W} / \mathbf{K}\right)$ \\
\hline VAC-01 & 1.44 (closed membrane) & 21.2 & 2.05 \\
\hline VAC-02 & 0.70 & 0.80 & 0.80 \\
\hline VAC-03-k & 1.78 & 0.42 & 0.91 \\
\hline VAC-03-g & 4.00 & 0.51 & 1.51 \\
\hline
\end{tabular}

\section{Sensor Packaging}

The sensor chips are die bonded to transistor outline headers, depending on the chip size either on a TO39 or on a TO8 header. The heating/sensing resistor $R_{P}$ on the thermally isolated membrane and one of the reference resistors $R_{K}$ on the silicon rim of the chip are connected to the pins by wire bonding. A metallic cap with a small opening $\left(\approx 1 \mathrm{~mm}^{2}\right)$ for pressure exchange is fixed on the header by high-current welding. Figure 3 shows the geometrical dimensions of sensors mounted in TO39 packages.

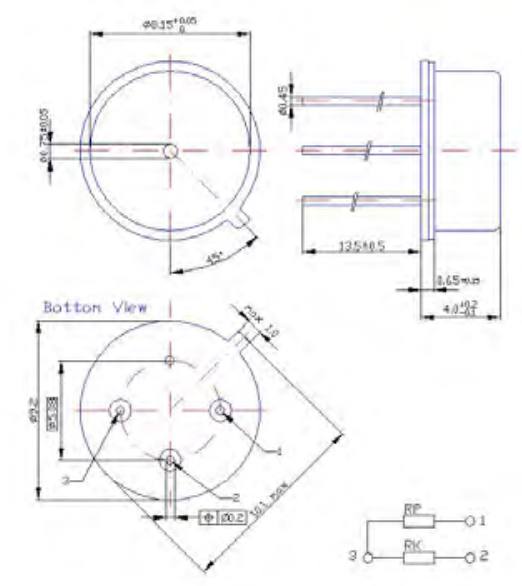

Fig. 3a: Sensor drawing with pin dimensions

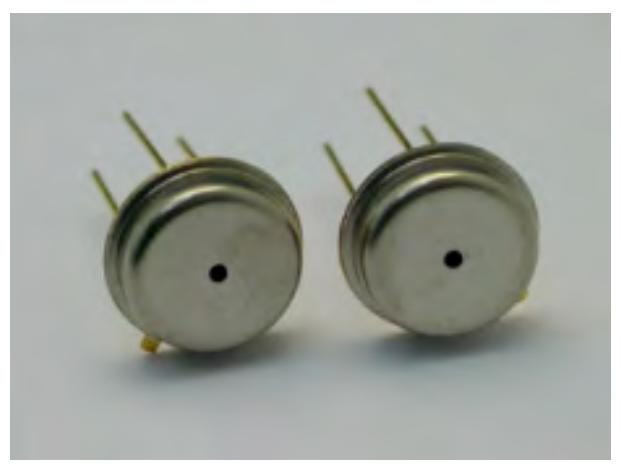

Fig. 3b: Picture of sensors in TO39 housings

Based on the simulation models and the successful confirmation of the theoretical results by the measurements of sensor characteristics, further chips for specific pressure ranges can be designed and optimized. VAC-03 has been especially designed for high sensitivity between 1 mbar and $10^{-5}$ mbar. Pirani type sensors usually suffer from low sensitivity in the pressure range 10 mbar to $10^{3}$ mbar, since 
the mean free path of gas molecules becomes small compared to distances $\left(d_{1}, d_{2}\right)$ between heated surfaces and heat sinks. However, micromachining offers the chance to realize very small distances (in the $\mu \mathrm{m}$ range) between heated membranes and "cold" surrounding areas and thus for an increased pressure sensitivity in the rough vacuum and atmospheric pressure range. The fact that a chip at these vacuum pressures requires only small heated area and chip size is an additional advantage. This makes it easier to integrate such a small chip together with VAC-03 chips into the same housing. Figure 4 shows the chip type VAC-04, designed for high sensitivity between $1 \mathrm{mbar}$ and $10^{3} \mathrm{mbar}$, mounted together with a VAC-03 chip on a 12 pin TO8 header. The VAC-04 chip size amounts to $1 \times 1 \mathrm{~mm}^{2}$. Such combinations of VAC-03 and VAC-04 provide pressure-dependent signals from $10^{3}$ mbar down to $10^{-5}$ mbar by using the appropriate chip in the corresponding pressure range.

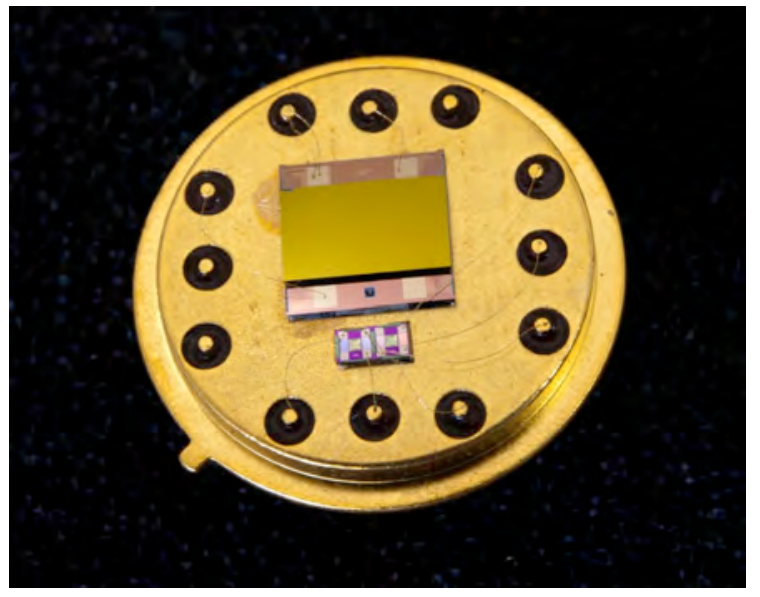

Fig. 4: Two vacuum sensors chips (VAC-03 and VAC-04) for different pressure ranges combined on a 12 pin TO8 header

\section{Sensor Performance}

The following curves (Fig 5 to Fig 7) show the signal voltages versus pressure $U(p)$ measured with different chip types. The Wheatestone bridge curcuits (Fig 2) have been zeroed at atmospheric pressure. A voltage supply $U_{B}=3 \mathrm{~V}$ and a corresponding heating power of about $200 \mu \mathrm{W}$ (dissipated in $R_{P}$ and $R_{K}$, respectively) have been applied for all measurements.

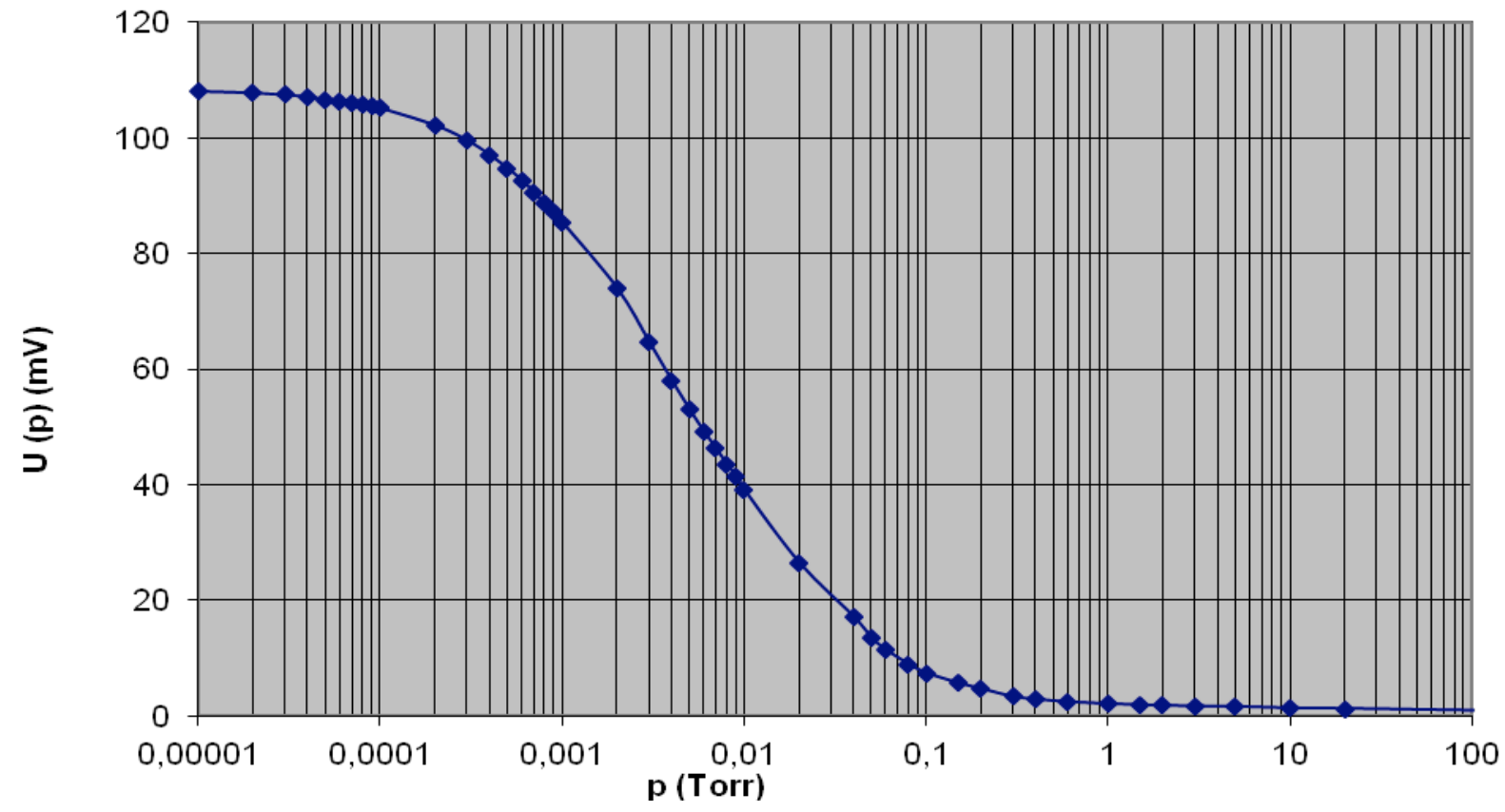

Fig. 5: Signal voltage $U(p)$ as function of pressure for chip type VAC-02 $\left(U_{B}=3 \mathrm{~V}\right)$ 


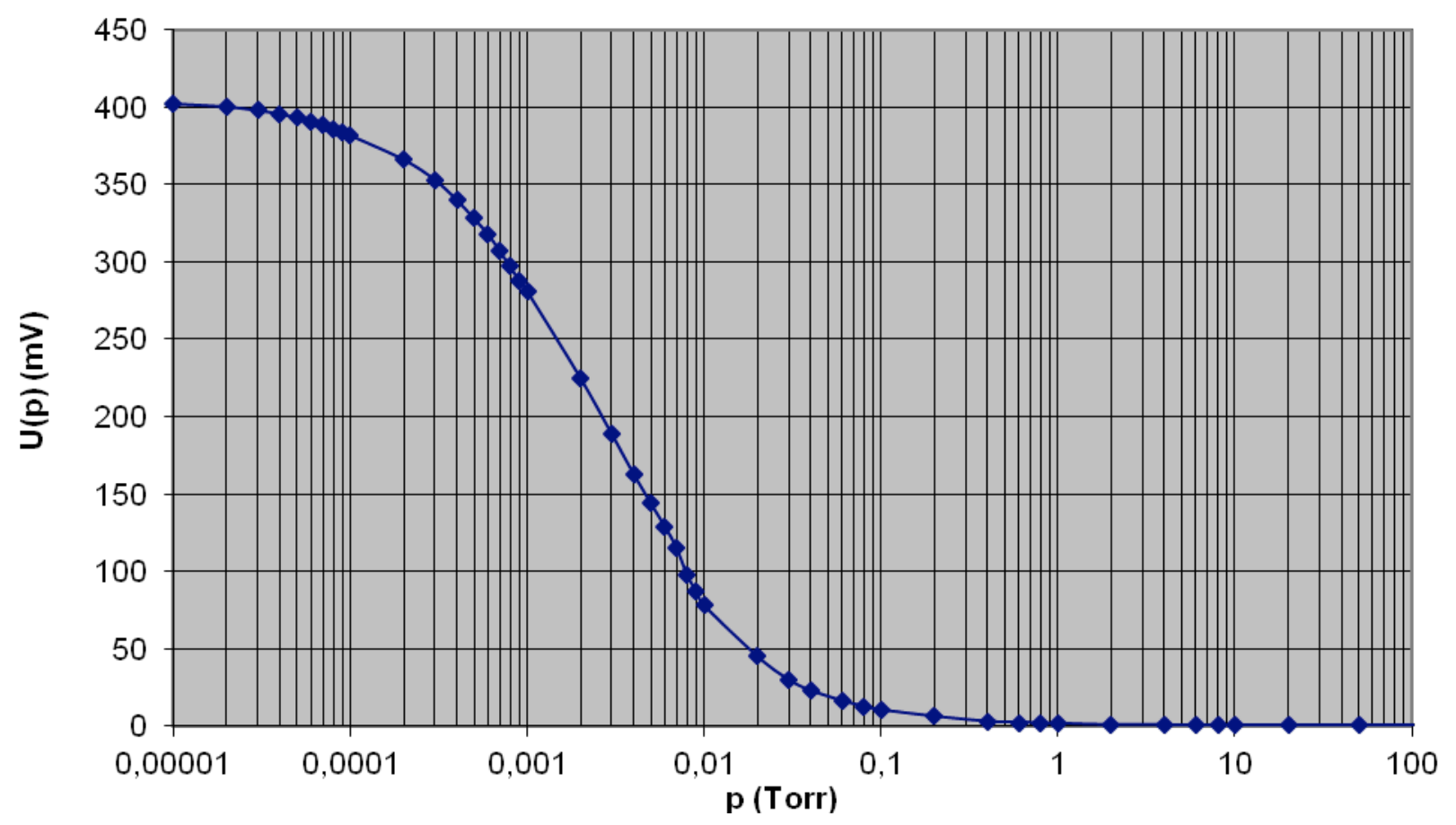

Fig. 6: Signal voltage $U(p)$ as function of pressure for chip type VAC-03-g $\left(U_{B}=3\right.$ V)

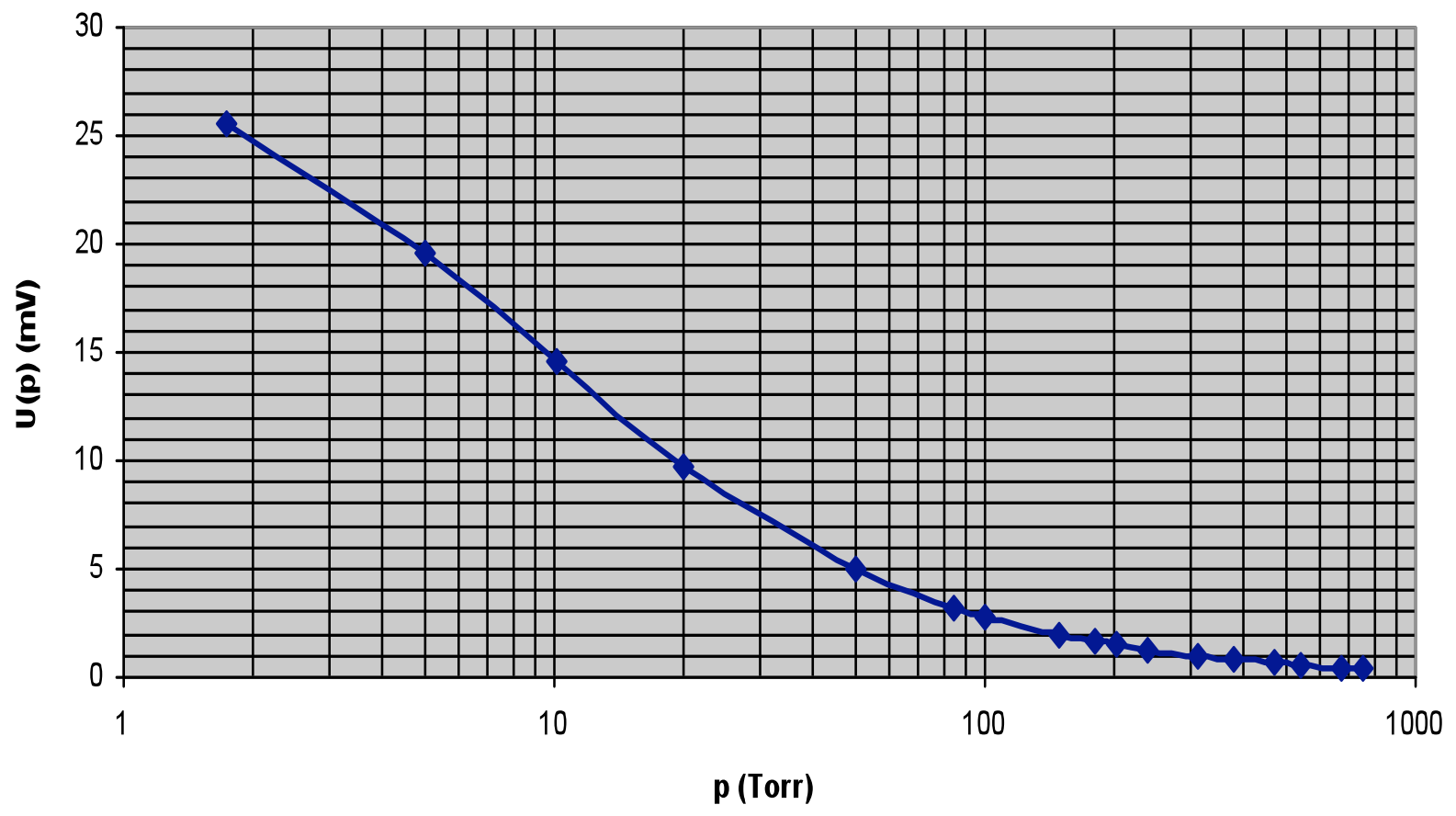

Fig. 7: Signal voltage $U(p)$ as function of pressure for chip type $V A C-04\left(U_{B}=3 V\right)$

\section{Temperature Drift}

Besides gas pressure the resistances $R_{P}\left(T_{p}\right)$ and $R_{K}\left(T_{0}\right)$ are also influenced by the drift of the ambient temperature $T_{0}$. For constant voltage supply $U_{B}$ and constant pressure $p$ an increase of $T_{0}$ leads to a decrease of the signal voltage $U\left(p, T_{0}\right)$ due to increasing thermal conductivity of gases and increasing radiation conductance. This pressure-independent signal drift has to be compensated by appropriate modifications of the readout circuit or by electronic compensation of the drift characteristic [10].

A useful modification of the Wheatstone bridge circuit to reduce the signal drift $U\left(p, T_{0}\right)$ is shown in Fig 8 . An external resistor $R_{\text {ext }}$ with a temperature coefficient $\beta=0$ is additionally integrated in the circuit. 
Efficient compensation effects can be observed, if $R_{K}\left(T_{0}\right) \approx R_{\text {ext }} \approx 2 R_{P}\left(T_{0}\right)$. By smart adaptation of the resistance and $B$ values the undesirable signal drift of the bridge output voltage can be significantly reduced.

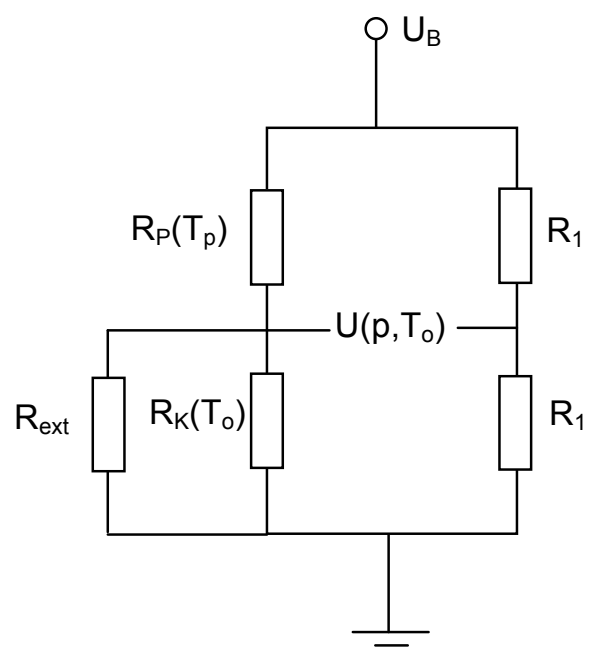

Fig 8: Wheatstone bridge circuit with external resistor $R_{\text {ext }}$ (temperature coefficient $\beta=0$ ) for temperature compensation of $U\left(p, T_{0}\right)$

\section{Summary}

Miniaturized Pirani type vacuum sensors have been manufactured using thin film heating/sensing resistor structures on thin suspended membranes. Thermal simulations have been performed to analyze the various terms that contribute to the thermal conductance between heated resistor and surroundings. Based on these theoretical results new designs have been realized that minimize the pressureindependent terms and thus maximize the pressure sensitivity of the chips.

Deposition parameters, microlithography and micromachining processes have been successfully adapted to fabricate chips on 4-inch (100) oriented silicon wafers. Various chip types are available, which have been optimized with respect to sensitivity for the pressure range $10^{3}$ mbar to 1 mbar (VAC-04) and the pressure range 1 mbar to $10^{-5}$ mbar (VAC-03). Both chip types can be integrated into the same TO housing. Thus we have built a new generation of miniaturized vacuum sensors that can detect vacuum pressure over 8 orders of magnitude.

The small size of the sensors together with the wide range of sensitivity will open new application fields for this MEMS type Pirani sensors.

\section{References}

[1] Wutz, Handbuch Vakuumtechnik, K. Jousten (Hrsg.), Vieweg 2006

[2] A.W. van Herwaarden, P.M. Sarro, Double-Beam Integrated Thermal Vacuum Sensor, Jour. of Vacuum Science and Technology 5 (1987) 2454-2457

[3] F. Völklein, W. Schnelle, A vacuum microsensor for the low vacuum range, Sensors and Materials, Vol. 3 (1991), 41-48

[4] P. K. Weng, J.-S. Shie, Rev. Sci. Instr., Vol. 65 (1994), 492-499

[5] O. Wenzel, C.K. Bak, The MicroPirani ${ }^{\mathrm{TM}}$ : a solid-state vacuum gauge with wide range, Vakuum in Forschung und Praxis 4 (1998), 298-301

[6] Patentschrift CN101256105(März 2008), J. Zhang, Y. Jin, J. Zhou: Monocrystalline silicon transverse miniature MEMS Pirani meter and preparation method thereof

[7] Patentschrift JP2007147344 (Juni 2007), K. Mitsutera: Thin film Pirani vacuum sensor and vacuum measuring device

[8] USPatent 7087451B2 (Aug. 2006), L.R. Arana, Y.L. Zou, J. Heck: Microfabricated hot wire vacuum sensor

[9] EP 94120290.5 (Dec. 1994), F. Völklein, J. Schieferdecker: Mikrovakuumsensor mit erweitertem Empfindlichkeitsbereich

[10] USPatent 7331237B2 (Feb. 2008), M. Borenstein: Technique for improving Pirani gauge temperature compensation over its full pressure range 